\title{
Hyperinsulinemia/euglycemia therapy (HIET) for the management of severe amlodipine toxicosis in a cat
}

\author{
Audrey Tinsman ${ }^{1}$ and Tara Bellis ${ }^{2}$ \\ ${ }^{1}$ Bluepearl Veterinary Partners \\ ${ }^{2}$ BluePearl Veterinary Specialists
}

May 3, 2021

\begin{abstract}
Calcium channel blocker overdose is encountered in human and veterinary medicine. At toxic levels these medications alter glucose metabolism, vascular tone, and cardiac function. Hyperinsulinemia/euglycemia therapy is a cardioprotective treatment used in human medicine, but rarely documented in veterinary medicine. This is the first report of HIET in a cat.
\end{abstract}

\section{Introduction}

Calcium-channel blockers (CCBs) have been used for over 40 years in veterinary medicine as both antihypertensive and anti-arrhythmic medications ${ }^{1}$. Three main categories of CCBs are commercially available in the United States for veterinary use: phenylalkylamines such as verapamil, benzothiazepines such as diltiazem, and dihydropyridines such as amlodipine. All are defined by their ability to block the long-lasting L-type calcium channels found primarily in the cardiac, smooth and skeletal muscle ${ }^{1}$. Calcium channels are vital for conduction of electric impulses across the sinoatrial and atrioventricular nodes of the heart. By inhibiting calcium influx, CCBs decrease atrioventricular conduction and slow the heart rate. In contractile cells such as vascular smooth muscle cells and myocytes, calcium channel blockade reduces cytosolic calcium levels and calcium-induced calcium release from the sarcoplasmic reticulum ${ }^{2}$. This leads to reduced cardiac inotropy and vasodilation, particularly in vascular beds with a high resting tone such as arteries. In addition, there is a dose-dependent response to CCBs within the systemic resistance vessels ${ }^{3}$. Other tissues affected by CCBs include the pancreas, pulmonary parenchyma, and central nervous system ${ }^{1}$. Blockade of L-type calcium channels in the pancreas causes decreased insulin release by the $\beta$-islet cells and restricts tissue uptake of glucose by altering sensitivity to insulin ${ }^{1}$. Under normal aerobic conditions, myocardial cells oxidize free fatty acids as the main energy substrate. In hypoperfused or hypoxic states, myocardial cells utilize glucose as their main substrate. In patients with CCB toxicosis, hypoinsulinemia, insulin resistance, and hypotension lead to reduced glucose delivery. That combined with an increased tissue demand causes a relative negative glucose balance leading to impaired inotropy and myocardial dysfunction ${ }^{4}$.

Calcium channel blocker toxicity causes an exaggeration of therapeutic effects including hypotension, bradycardia and bradyarrhythmias. Additional clinical signs include gastrointestinal upset, hypothermia, central nervous system depression, noncardiogenic pulmonary edema, hyperglycemia, hypokalemia, hyponatremia, metabolic acidosis, often coupled with hyperlactatemia, and, rarely, stimulatory signs such as agitation or seizures $^{1}$. First-line treatments for CCB toxicosis include decontamination and supportive care; secondline treatments include calcium supplementation, insulin-glucose infusions, vasopressors/catecholamines, glucagon, intralipid therapy, and external pacing ${ }^{1}$.

Hyperinsulinemia/euglycemia therapy (HIET) is described in the human literature and infrequently described in the veterinary literature as a therapy for CCB toxicity. It consists of a high-dose regular insulin infusion 
coupled with a glucose infusion ${ }^{6}$. Intracellular transport of glucose in cardiac and skeletal muscle is greatly enhanced by insulin providing an ongoing source of energy for cardiac contraction. In high concentrations, insulin affects several intracellular pathways that increase the inotropy of cardiac myocytes and alter calcium handling ${ }^{2}$. Additional benefits of HIET include increased cyclic AMP levels through phosphodiesterase III inhibition, resulting in increased calcium influx ${ }^{6}$. There is evidence that HIET increases endothelial nitric oxide synthase (eNOS) activity which in turn decreases capillary resistance and improves overall tissue perfusion $^{5}$. There is one published case report in the veterinary literature describing HIET for the treatment of CCB toxicosis in a dog. Maton et al describe the successful use of HIET in the treatment of diltiazem toxicosis $^{7}$. To the authors' knowledge this is the first case report describing the use of HIET for the treatment of CCB toxicosis in a cat.

\section{Case summary}

A 15-year-old female spayed Siamese cat presented for amlodipine toxicosis. Two months prior, the cat was treated by her primary veterinarian for a urinary tract infection and diagnosed with hypertension. She was started on telmisartan 6.5mg PO q24h (Semintra, Boehringer Ingelheim Vetmedica, Inc., St. Joseph, MO) and transitioned onto amlodipine 2 weeks prior to hospitalization. For the 5 days leading up to presentation the cat received $2.5 \mathrm{mg}$ instead of $0.625 \mathrm{mg}$ per os every 24 hours. The cat had known pre-existing renal disease which was managed with a prescription diet and stool softener (Miralax, Bayer Healthcare LLC, Whippany, NJ; 1/8tsp per os ever 12 hours).

The cat presented to the authors' hospital for decreased appetite and water intake for 24 hours and lethargy for several days. The owner reported a single episode of vomiting the night before. Amlodipine was last given that morning. Upon presentation (day one), the cat was dehydrated with a prolonged skin tent and weak femoral pulses. She had a decreased axillary temperature $\left(35.5^{\circ} \mathrm{C} ; 38.1-39.2^{\circ} \mathrm{C}\right)^{8}$ and borderline bradycardia (160 beats per minute). She was weakly ambulatory and quiet. Systolic blood pressure (SBP) as measured by Doppler ultrasonography (Doppler flow detector model 811-B, Parks Medical Electronics) was low at $70 \mathrm{mmHg}(110-132 \mathrm{mHg})^{9}$. An intravenous (IV) catheter was placed, and a minimum database was performed, which identified mild anemia and hyperproteinemia (see Table 1). Spot blood glucose (sBG), (AlphaTRAK 2, Abbott Animal Health, Abbott Park, IL) was high at $14.37 \mathrm{mmol}$;/L $(259 \mathrm{mg} / \mathrm{dl}$; RI [3.72$9.32 \mathrm{mmol} / \mathrm{L}])^{10}$. A venous blood gas (vBG; RAPIDPoint 500 System, Siemens Healthineers, Malvern, PA) revealed a metabolic acidosis with hyperlactatemia (see Table 1). A chemistry panel (Catalyst One, IDEXX Laboratories Inc, Westbrook, ME) revealed azotemia and hyperamylasemia (see Table 2). A 10ml/kg IV crystalloid bolus of LRS (Lactated Ringer's Solution; Dechra Veterinary Products, Overland Park, KS) was administered. Systolic BP improved to $92 \mathrm{mmHg}$. A $90 \mathrm{mg} / \mathrm{kg}$ calcium gluconate (Calcium Gluconate Injection USP 100mg per mL, HF Acquisition Co LLC, DBA HealthFirst) bolus diluted 1:1 with $\mathrm{NaCl} 0.9 \%(0.9 \%$ Sodium Chloride Injection USP, B.Braun Medical, Irvine, CA) was given intravenously over 20 minutes. The cat was started on intravenous fluid therapy with $\mathrm{NaCl} 0.9 \%$ and was administered maropitant citrate (Cerenia 10mg/ml, Zoetis Inc, Kalamazoo, MI; $1 \mathrm{mg} / \mathrm{kg}$ IV q24h).

The following morning (day 2), the cat was transferred to the Critical Care service. Systolic blood pressure was $80 \mathrm{mmHg}$ and rectal temperature was $32.9^{\circ} \mathrm{C}\left(38.1-39.2^{\circ} \mathrm{C}\right)^{8}$. Repeat bloodwork revealed persistent hyperglycemia with a sBG of $23.87 \mathrm{mmol} / \mathrm{L}(430 \mathrm{mg} / \mathrm{dl} \text {; RI }[3.72-9.32])^{10}$. Serum ketones were negative (Precision Xtra, Abbott Animal Health, Abbott Park, IL). A vBG showed a worsening metabolic acidosis with improving hyperlactatemia (see Table 1). A CBC (Procyte Dx Hematology Analyzer, IDEXX Laboratories Inc, Westbrook, ME) showed a non-regenerative anemia and neutrophilia (see Table 2). One unit (1U) regular insulin (HumulinR, Lilly USA LLC, Indianapolis, IN) was administered IV. Two further doses (90mg/kg and $150 \mathrm{mg} / \mathrm{kg}$ respectively) of calcium gluconate diluted $1: 1$ with $\mathrm{NaCl} 0.9 \%$ were given IV. Bradycardia worsened to 90 beats per minute and a single dose of atropine sulfate (Atropine Sulfate Injection 1/120 Grain, Med-Pharmex, Pomona, CA) $0.04 \mathrm{mg} / \mathrm{kg}$ IV was given with no effect. A calcium gluconate continuous rate infusion (CRI) was started at $0.05 \mathrm{mcg} / \mathrm{kg} / \mathrm{h}$. Bradycardia persisted and an electrocardiogram (cECG; MD9000vet, Meditech Equipment Co., Ltd) identified intermittent absence of P-waves. A point-of-care thoracic ultrasound showed scant pleural effusion and markedly decreased cardiac contractility. Intravenous 
fluid therapy was discontinued and a dobutamine CRI $(2.5-3.75 \mathrm{mcg} / \mathrm{kg} / \mathrm{min}$; DOBUTamine Injection USP, Hospira Inc., Lake Forrest IL) was started; calcium gluconate was discontinued. Intralipid emulsion therapy (ILE; Intralipid $20 \%$ Baxter Healthcare Corporation, Deerfield, IL) was administered: $0.5 \mathrm{mls} / \mathrm{kg}$ IV bolus followed by $0.25 \mathrm{mls} / \mathrm{kg} / \mathrm{min}$ for 1 hour. Hypothermia worsened to $33.2^{\circ} \mathrm{C}\left(38.1-39.2^{\circ} \mathrm{C}\right)^{8}$ despite heat support and the cat's mentation deteriorated to dull. Serial vBGs showed a worsening metabolic acidosis and hypokalemia (see Table 1). Crystalloid fluid therapy was restarted at a rate of $5 \mathrm{ml} / \mathrm{hr}$ with potassium supplementation (Potassium Chloride injection $2 \mathrm{mEq} / \mathrm{ml}$, Hospira Inc Lake Forest, IL) at $80 \mathrm{meq} / \mathrm{L}$. High-dose insulin/euglycemia therapy (HIET) was initiated with a bolus of $1 \mathrm{U} / \mathrm{kg}$ IV regular insulin and a bolus of $1 \mathrm{~g} / \mathrm{kg}$ dextrose (50\% dextrose injection, USP $25 \mathrm{~g} / 50 \mathrm{ml}$ Hospira Inc., Lake Forest IL) IV followed by a regular insulin CRI at $1 \mathrm{U} / \mathrm{kg} / \mathrm{h}$ for the first hour with a rate decrease to $0.5 \mathrm{U} / \mathrm{kg} / \mathrm{h}$ as of the second hour. A $5 \%$ dextrose CRI was administered concurrently. Within 5-10 minutes of starting HIET, there was a notable improvement in the patient's blood pressure, heart rate and temperature. After 2 hours of therapy, blood pressure was $112 \mathrm{mmHg}$, heart rate was 140 beats per minute with normal sinus rhythm, and temperature was $36.7^{\circ} \mathrm{C}$. Serum potassium increased (see Table 1) and potassium supplementation was decreased from 80 to $60 \mathrm{mEq} / \mathrm{L}$. Hypoglycemia $(\mathrm{BG}<3.72 \mathrm{mmol} / \mathrm{L}$ ) was identified five times during HIET; and was treated with intermittent dextrose boluses $(0.5 \mathrm{~g} / \mathrm{kg} \mathrm{IV})$ and an increase in dextrose supplementation to $7.5 \%$. Insulin therapy was discontinued after approximately 3 hours due to persistent hypoglycemia. At that time the cat was ambulatory and interactive. Point-of-care ultrasound showed a marked improvement in cardiac contractility. Overnight dextrose supplementation was decreased then discontinued as euglycemia was maintained. Crystalloid fluids were continued at $5 \mathrm{mls} / \mathrm{hr}$ with $60 \mathrm{mEq} \mathrm{KCl} / \mathrm{L}$ supplementation. Temperature normalized and heat support was discontinued. Heart rate remained $>150$ beats per minute and blood pressure remained between $90 \mathrm{mmHg}$ and $132 \mathrm{mmHg}$ on a dobutamine CRI at $3.75 \mathrm{mcg} / \mathrm{kg} / \mathrm{min}$.

Early the next day (Day 3), the cat became hypotensive with a decrease in SBP to $80 \mathrm{mmHg}$. A CRI of norepinephrine (Levophed TM, Norepinephrine Bitartrate Injection USP, Hospira Inc., Lake Forest, IL) was started at $0.1 \mathrm{mcg} / \mathrm{kg} / \mathrm{min}$ and titrated up to $0.2 \mathrm{mcg} / \mathrm{kg} / \mathrm{min}$. Mild respiratory effort was noted and oxygen therapy at FiO2 0.4 (Snyder MFG.CO Intensive Care Unit) was provided. Point-of-care ultrasound revealed an increase in the volume of pleural effusion. Based on progression of clinical signs and financial constraints, the owners elected humane euthanasia.

\section{Discussion:}

This is the first case report describing hyperinsulinemia/euglycemia therapy for the treatment of amlodipine toxicosis in a cat. Calcium channel blockers are rapidly and completely absorbed after ingestion ${ }^{10}$. Amlodipine has the highest bioavailability and volume of distribution in its class ${ }^{11}$, is highly protein bound and hepatically metabolized prior to renal excretion. The elimination half-life is potentially the longest of any CCB at 30 hours in the dog, with a range of 30-60 hours reported in the cat ${ }^{1}$. Time to peak plasma concentration for immediate release forms is 20-45 minutes. The minimum toxic dose has not been established in humans or animals, however signs of toxicosis at therapeutic doses have been noted in all classes of $\mathrm{CCB}^{1}$. Amlodipine toxicosis is the most commonly reported dihydropyridine toxicosis in animals, accounting for $83.2 \%$ of the cases reported to the ASPCA APCC from 2000 to $2017^{1}$. The clearance of CCBs is likely prolonged in disease states such as congestive heart failure, cardiomyopathy, or hypotensive overdose which result in altered hepatic perfusion ${ }^{12}$. Standard dosage is typically $0.625-1.25 \mathrm{mg}$ per cat ${ }^{8}$. Little is known about the cumulative effects of amlodipine, but signs of toxicosis in people may persist for up to 10 days, with mortality in symptomatic cases as high as $21 \%^{13}$.

Decontamination and limitation of toxin absorption is the first line of treatment if ingestion occurred within 2 hours of presentation ${ }^{1}$. Emesis was not attempted in the cat reported here due to the chronicity of the exposure. Activated charcoal was not administered due to the concern for aspiration. A warm water enema could have been performed to facilitate evacuation of intestinal contents ${ }^{1}$. Calcium gluconate is recommended to overcome inhibited calcium channels and promote inotropy, but response to treatment is inconsistent in severe intoxications ${ }^{5}$. An exact dose of calcium is not described but a CRI titratable to a desirable blood pressure and heart rate has been suggested ${ }^{10}$. The administration of calcium gluconate in this case led 
to ionized hypercalcemia without any clear benefit. Atropine may increase heart rate and improve cardiac output, however the effect is often transient ${ }^{2}$. Furthermore, the resulting increase in myocardial oxygen demand can lead to myocardial injury when systemic and coronary hypotension are present ${ }^{14}$. Atropine had no positive effect in the present case. Vasopressors can increase SBP by overriding the vasodilatory effects of amlodipine, but the resulting increase in systemic vascular resistance can lead to decreased cardiac output and altered perfusion of vascular beds ${ }^{5}$. Treatment with adrenergic drugs may yield a poor response in patients with moderate to severe overdoses ${ }^{10}$. Glucagon is a pancreatic peptide and cardiac inotrope ${ }^{1}$. It increases cardiac output, lowers pulmonary arterial pressure, and pulmonary vascular resistance without any systemic vascular effects ${ }^{15}$. Several animal models evaluating glucagon for the treatment of CCB toxicosis have shown an increase in HR and cardiac output, as well as AV block reversal, however no effect on MAP or survival was observed ${ }^{10}$. Glucagon was not used in the case reported here due to lack of availability, and associated cost.

The use of lipid emulsion therapy (ILE) has been described in the treatment of toxicities from substances with a high lipid solubility ${ }^{16}$, including $\mathrm{CCBs}^{7}$. The mechanism of action of ILE is incompletely understood. One theory is that ILE sequesters the lipophilic drug in a 'lipid sink' reducing availability and promoting clearance through metabolism of drug-containing chylomicrons ${ }^{16}$. Another theory for the benefit of ILE in cardiotoxic drugs is that increased availability of free fatty acids may prevent the myocardium from switching to glucose as its preferred energy substrate ${ }^{16}$. Furthermore, long chain fatty acids may activate myocyte calcium channels resulting in increased calcium influx ${ }^{16}$. The inhibition of amlodipine-induced cardiomyoblast apoptosis using ILE has been demonstrated in a murine model ${ }^{17}$. Complications associated with ILE are infrequent but include microbial or particulate contamination of the lipid product, pancreatitis, hyperlipidemia, or an allergic-type reaction to the infusion ${ }^{16}$. For the cat reported here HIET was started shortly after ILE so the role of ILE alone cannot be evaluated. No complications were observed.

There are three major mechanisms by which HIET is believed to improve survival following CCB overdose: (1) increased inotropy, (2) increased intracellular glucose transport, and (3) vasodilation ${ }^{5}$. High-dose insulin increases coronary blood flow without increasing oxygen demands, contrary to catecholamines ${ }^{6}$. Insulin also increases eNOS activity leading to systemic, coronary, and pulmonary vasodilation ${ }^{5}$. Cardiogenic shock is characterized by altered microvascular perfusion and a failure to supply capillary beds and the surrounding tissues with metabolic substrate. By increasing eNOS activity, insulin enhances microvascular hemodynamics improving tissue perfusion, decreasing afterload, and increasing cardiac output ${ }^{5}$. In cell cultures, supraphysiologic doses of insulin are required to increase eNOS activity above basal concentrations, which may explain why hyperinsulinemia is needed to elicit these beneficial vascular effects ${ }^{5}$.

A study by Kline et al compared normal saline, epinephrine, glucagon, calcium chloride and HIET for the treatment of verapamil toxicosis in dogs. Dogs that received HIET had an increased survival rate, improved maximum elastance, left ventricular end diastolic pressure, ventricular relaxation, and coronary artery blood flow ${ }^{18}$. An experimental study by Kerns et al showed that insulin-treated anesthetized dogs following propranolol-induced cardiomyopathy had improved cardiodynamics and hemodynamics, as well as increased myocardial glucose uptake compared to dogs treated with glucagon or epinephrine. Survival was significantly higher in the insulin group compared with the glucagon and epinephrine groups ${ }^{15}$. Krukenkamp et al. induced myocardial depression in dogs using propranolol and found $80 \%$ reversal following HIET, as well as a statistically significant increase in peak blood pressure without changing myocardial oxygen consumption ${ }^{19}$. Beta-blocker overdose is often used in experimental models to extrapolate data which can then be applied to clinical CCB and beta-blocker toxicities as both drug classes result in blockade of calcium flux through the L-type calcium channels ${ }^{20}$. Two experimental porcine models evaluated the use of vasopressors and HIET in propranolol-induced cardiogenic shock ${ }^{21,22}$. The first study found that pigs treated with insulin and dextrose had a consistent improvement in cardiac output over the duration of treatment compared with the pigs treated with vasopressors alone, which had a progressive worsening of cardiac output ${ }^{21}$. The authors concluded that higher insulin doses may be required in the presence of vasopressors to overcome increased SVR and decreased cardiac output ${ }^{21}$. The second study found that patients treated with norepinephrine after maximizing high-dose insulin therapy had improved brain tissue oxygenation compared to HIET alone ${ }^{22}$ 
and that patients with profound hypotension $(<55 \mathrm{mmHg})$ could benefit from vasopressors after maximizing HIET.

There is strong evidence supporting the benefits of HIET in humans with CCB toxicosis, however there is little clinical evidence in the veterinary literature to support its use. One case report by Maton et al describes the use of HIET in conjunction with ILE to treat diltiazem toxicosis in a dog. In that case, HIET was initiated after clinical signs did not improve despite decontamination, calcium gluconate, dopamine CRI, glucagon, insulin pulse therapy with dextrose supplementation at $5 \%$ and ILE. Dextrose supplementation was increased to $10 \%$ at initiation of HIET and a regular insulin CRI was started at $1 \mathrm{U} / \mathrm{kg} / \mathrm{h}$. Within one hour of starting the insulin CRI clinical signs improved. The dog received a total of 20 hours of ILE therapy and 17 hours of insulin CRI and dextrose CRI which were slowly weaned. The patient was discharged without long-term complications ${ }^{7}$

\section{Conclusion}

In the cat reported here, a notable response to HIET was appreciated as evidenced by an increase in temperature, heart rate, blood pressure and contractility within 15 minutes of initiating therapy. The dosing recommendation for animals is a $1 \mathrm{U} / \mathrm{kg}$ insulin bolus followed by a $1 \mathrm{U} / \mathrm{kg} / \mathrm{h}$ CRI for 1 hour, followed by 0.5 $\mathrm{U} / \mathrm{kg} / \mathrm{hr}$ until resolution of clinical signs ${ }^{10}$. There is no set protocol for timing of discontinuation of HIET, however the goal of therapy is hemodynamic stability. Based on previous reports and human guidelines, prolonged therapy may be needed. The primary complications associated with HIET are hypoglycemia and hypokalemia; both of which can be anticipated, monitored and addressed. The initiation of vasopressor therapy in the context of amlodipine toxicosis should be approached with caution and considered after initiation and optimization of HIET. The authors also recommend monitoring of magnesium and phosphorus, which have a complex interplay with insulin and are vital for myocardial cellular activity. The wide availability of insulin and dextrose, low expense, and minimal adverse event profile make HIET an attractive therapeutic option for CCB toxicosis. Although the outcome was unsatisfactory in this case, the authors feel that the response to HIET supports the use of this therapy in cats with CCB toxicosis.

1. Hayes CL. An Update on Calcium Channel Blocker Toxicity in Dogs and Cats. Vet Clin North Am Small Anim Pract . 2018;48(6):943-957.

2. Kittleson, Mark D K Richard D. Small Animal Cardiovascular Medicine .

3. Costello M, Syring RS. Calcium channel blocker toxicity. J Vet Emerg Crit Care . 2008;18(1):54-60.

4. Depre Christophe, Vanoverschelde Jean-Louis J., Taegtmeyer Heinrich. Glucose for the Heart. Circulation . 1999;99(4):578-588.

5. Engebretsen KM, Kaczmarek KM, Morgan J, Holger JS. High-dose insulin therapy in beta-blocker and calcium channel-blocker poisoning. Clin Toxicol Phila Pa . 2011;49(4):277-283.

6. Lheureux PE, Zahir S, Gris M, Derrey A-S, Penaloza A. Bench-to-bedside review: Hyperinsulinaemia/euglycaemia therapy in the management of overdose of calcium-channel blockers. Crit Care . $2006 ; 10(3): 212$.

7. Maton BL, Simmonds EE, Lee JA, Alwood AJ. The use of high-dose insulin therapy and intravenous lipid emulsion to treat severe, refractory diltiazem toxicosis in a dog. J Vet Emerg Crit Care San Antonio Tex 2001 . 2013;23(3):321-327.

8. Kahn CM, ed. The Merck Veterinary Manual . 9. Aufl. Merck; 2005.

9. Payne JR, Brodbelt DC, Luis Fuentes V. Blood Pressure Measurements in 780 Apparently Healthy Cats. $J$ Vet Intern Med . 2017;31(1):15-21.

10. Silverstein DC, Hopper K, eds. Small Animal Critical Care Medicine . 2. ed. Saunders, Elsevier; 2015. 
11. Stopher DA, Beresford AP, Macrae PV, Humphrey MJ. The metabolism and pharmacokinetics of amlodipine in humans and animals. J Cardiovasc Pharmacol . 1988;12 Suppl 7:S55-59.

12. Holder T. Calcium channel blocker toxicosis. :4.

13. Zahed N-S, Hassanian-Moghaddam H, Zamani N. A Fatal Case of Amlodipine Toxicity Following Iatrogenic Hypercalcemia. Cardiovasc Toxicol . 2018;18(3):290-293.

14. Monteiro ER, Campagnol D, Parrilha LR, Furlan LZ. Evaluation of cardiorespiratory effects of combinations of dexmedetomidine and atropine in cats. J Feline Med Surg . 2009;11(10):783-792.

15. Kerns W, Schroeder D, Williams C, Tomaszewski C, Raymond R. Insulin Improves Survival in a Canine Model of Acute $\beta$-Blocker Toxicity.Ann Emerg Med . 1997;29(6):748-757.

16. Fernandez AL, Lee JA, Rahilly L, Hovda L, Brutlag AG, Engebretsen K. The use of intravenous lipid emulsion as an antidote in veterinary toxicology. J Vet Emerg Crit Care . 2011;21(4):309-320.

17. Ok S-H, Ahn SH, Kim H-J, et al. Lipid emulsion attenuates extrinsic apoptosis induced by amlodipine toxicity in rat cardiomyoblasts.Hum Exp Toxicol . Published online October 8, 2020:960327120964551.

18. Kline JA, Tomaszewski CA, Schroeder JD, Raymond RM. Insulin is a superior antidote for cardiovascular toxicity induced by verapamil in the anesthetized canine. J Pharmacol Exp Ther . 1993;267(2):744-750.

19. Krukenkamp I, Sørlie D, Silverman N, Pridjian A, Levitsky S. Direct effect of high-dose insulin on the depressed heart after beta-blockade or ischemia. Thorac Cardiovasc Surg . 1986;34(5):305-309.

20. Woodward C, Pourmand A, Mazer-Amirshahi M. High dose insulin therapy, an evidence based approach to beta blocker/calcium channel blocker toxicity. DARU J Pharm Sci . 2014;22(1):36.

21. Holger JS, Engebretsen KM, Fritzlar SJ, Patten LC, Harris CR, Flottemesch TJ. Insulin versus vasopressin and epinephrine to treat $\beta$-blocker toxicity. Clin Toxicol . 2007;45(4):396-401.

22. Katzung KG, Leroy JM, Boley SP, Stellpflug SJ, Holger JS, Engebretsen KM. A randomized controlled study comparing high-dose insulin to vasopressors or combination therapy in a porcine model of refractory propranolol-induced cardiogenic shock. Clin Toxicol . 2019;57(11):1073-1079.

23. Lindeman E, Ålebring J, Johansson A, Ahlner J, Kugelberg FC, Nordmark Grass J. The unknown known: non-cardiogenic pulmonary edema in amlodipine poisoning, a cohort study. Clin Toxicol Phila Pa . 2020;58(11):1042-1049.

24. Paige CF, Abbott JA, Elvinger F, Pyle RL. Prevalence of cardiomyopathy in apparently healthy cats. $J$ Am Vet Med Assoc . 2009;234(11):1398-1403.

25. Bachmann K, Kutter AP, Schefer RJ, Marly-Voquer C, Sigrist N. Determination of reference intervals and comparison of venous blood gas parameters using standard and non-standard collection methods in 24 cats. J Feline Med Surg . 2017;19(8):831-840.

\section{Hosted file}

tables case report.pdf available at https://authorea.com/users/411475/articles/520489hyperinsulinemia-euglycemia-therapy-hiet-for-the-management-of-severe-amlodipinetoxicosis-in-a-cat

\section{Hosted file}

Additional table case report.pdf available at https://authorea.com/users/411475/articles/ 520489-hyperinsulinemia-euglycemia-therapy-hiet-for-the-management-of-severe-amlodipinetoxicosis-in-a-cat 